\title{
Solving stochastic multiobjective vehicle routing problem using probabilistic metaheuristic
}

\author{
Asmae Gannouni ${ }^{1,2, \star}$, Rachid Ellaia ${ }^{1}$, and El-Ghazali Talbi ${ }^{2}$ \\ ${ }^{1}$ LERMA, Mohammadia School of Engineering, Mohammed V University Rabat, Morocco \\ ${ }^{2}$ CRIStAL/Dolphin, University Lille 1
}

\begin{abstract}
In this paper we propose a new metaheuristic algorithm for solving stochastic multiobjective combinatorial optimization(SMOCO) problems. Indeed, we find that the various initiatives that have been launched recently on this subject, they propose the classical metaheuristics to solve a stochastic multi-objective problems, but when the stochasticity effects is not taken into account, the choice of an arbitrary value leading to a particular configuration and a high loss of information. To conserve the stochastic nature of SMOCO problems, the pareto ranking should be defined on the random objectives functions directly rather than converted deterministic objectives. From these considerations, the scope of this research should consist of: (i)Proposed novel methodology of stochastic optimality for ranking objective functions characterized by non-continuous and no closed form expression. This novel approach is based on combinatorial probability and can be incorporated in a multiobjective evolutionary algorithm. (ii)Provide probabilistic approaches to elitism and diversification in multiobjective evolutionary algorithms. Finally, The behavior of the resulting Probabilistic Multi-objective Evolutionary Algorithms (PrMOEAs) is empirically investigated on the multi-objective stochastic VRP problem.
\end{abstract}

\section{Introduction}

Many real-life optimization problems encountered in logistics, transportation, and Markets finance have several conflicts objectives under aleatory uncertainty to be satisfied, traditionally such problems were handled by converting the stochastic multiple objectives to deterministic multiple objectives using statistical aggregation: mean value, extreme value, variance..., then applying the classical metaheuristics [1], such as pareto evolutionary algorithms for generate a set of well-distributed pareto-optimal solutions [13]. Such an approach has many problems, the including the loss of significant trade-off information and the inability to search the true objective space because it is incapable to describe the relationship among stochastic(random) objectives. Personally speaking, to represent and conserve the stochasticity of original SMOCO problems, we should proposed other methodology of optimality under aleatory uncertainty. Therefore, the aim of this study is to provide a probabilistic definition, to dominance, elitism and diversification in multi-objective evolutionary algorithms.

Based on the literature, the authors have classified methods for solving stochastic multiobjective combinatorial optimization problems, into three specific categories based on the way the objective function is estimated: i)Deterministic approaches: have been focused on treating it in multiobjective design by avoiding or ignoring un-

\footnotetext{
^e-mail: asmaegannouni@gmail.com
}

certainty[18], then the solution of the stochastic multiobjective optimization problems is not essentially different from that of a deterministic multiobjective optimization problems, and exact(lerning method, random optimization methods, stochastic approximation) [5] or metaheuristic techniques(NSGAII, SPEAII,...) can be used $[4,15]$ easily. ii) Robust Approaches: In this categorization, researchers reduced the stochastic nature of the problem by using for example the expectation of random functions: sample average approximation [17], or pareto means, pareto variance, pareto Quantile,...[6,12], these approaches are similar to the deterministic approaches, pareto dominance is used after changing the methodologies of the evaluation. Finally, for (iii)Stochastic Approaches: only few techniques are available and are still in nascent $[11,16]$, this work is part of these Approaches, here the stochastic optimality is the major challenge. These approaches are well the resulting of hybridization between the probabilistic programming, simulation $[8,10]$ and metaheuristic algorithms.

The remainder of this paper is organized as follows: In section 2 , we investigate the formula of stochastic multiobjective optimization problems and we discusse the main concepts in multiobjective evolutionary algorithms that can be extended in stochastic context. Section 3, describes the design of Probabilistic approaches: probabilistic dominance, probability memory and diversification for practical use. He whole methodology is then illustrated on analytical test case: Multiobjective stochastic VRP in section 
4 , followed by conclusions and future prospects in Section 5 .

\section{Stochastic Multi-objective Optimization}

Stochastic multi-objective optimization problems[7] have been investigated thoroughly in the operations research community. We start this section by giving a general formula of SMOCO problems, we then expose general concepts that share these problems and the challenge that it entails. Then we present pareto multi-objective evolutionary algorithms which will be be extended on stochastic context[21-23] in the next section.

\subsection{Stochastic Multi-objective Optimization Problems}

In the stochastic multi-objective optimization problems, two or more usually conflicting stochastic(uncertain) objectives are required to be optimized simultaneously, several types of formulations for SMOCO problems are possible, to be more specific let us define the problem in terms of the minimization that motivated the present research:
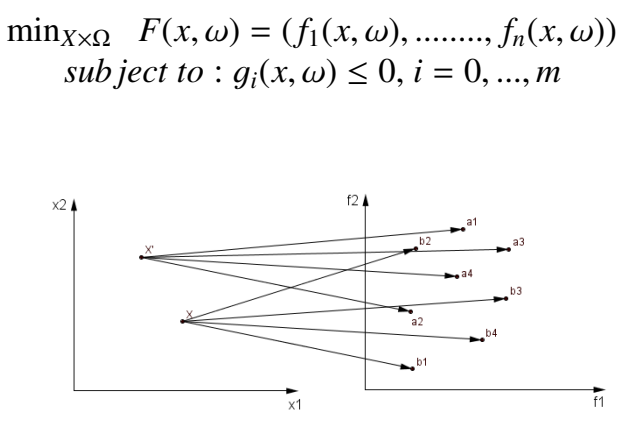

Figure 1. Evaluations of two individuals $x$ and $x^{\prime}$

Where $f_{i}(x, \omega)(i=1, \ldots, n)$ (for each fixed $x$ is the set of decision variables from the decision space $X$ ), are Borel functions(random variables) defined on a probability space $(\Omega, \mathcal{F}, \mathbb{P})$ and $\omega$ is an element of the sample space $\Omega$. Each $\omega$ determines a specific realization (a scenario)of the random variable $f_{i}(x,$.$) . (x$ it is called an individual in evolutionary multiobjective optimization algorithms MOEAs). The probability space $(\Omega, \mathcal{F}, \mathbb{P})$ is defined as follows: - $\Omega$ is the space of elementary events.

- $\mathcal{F}$ is the $\sigma$-algebra constructed from all subsets of $\Omega$.

- $\mathbb{P}$ is a probability measure defined on the measurable space $(\Omega, \mathcal{F})$.

From this formulation of SMOCO problems, each objective function can yield different values for the same individual from time to time (Figure.1), concerning the multiobjective part, we focus on the solution concept of determining pareto-optimal solutions, under this approach there are three possible relationships between the exact solutions:

Definition.1 (Weak Dominance) $x$ weakly dominates $x^{\prime}$ denoted by $x \leq x^{\prime}$ if $f_{i}(x) \leq f_{i}\left(x^{\prime}\right), \forall i \in\{1, \ldots, m\}$. Definition.2 (Strong Dominance) $x$ strongly dominates $x^{\prime}$ denoted by $x \leq x^{\prime}$ if $f_{i}(x)<f_{i}\left(x^{\prime}\right), \forall i \in\{1, \ldots, m\}$.

Definition.3 (Incomparable) $x$ is incomparable with $x^{\prime}$ denoted by $x \sim x^{\prime}$ if $f_{i}(x)>f_{i}\left(x^{\prime}\right), \exists i \in\{1, \ldots, m\}$ and $f_{j}(x)>f_{j}\left(x^{\prime}\right) \exists \mathrm{j} \in\{1, \ldots, m\}$.

Definition.4 (Nondominated vector) An objective vector $F(x)$ is said to be nondominated iff there does not exist another objective vector $F\left(x^{\prime}\right)$ such that $x^{\prime}>x$.

Definition.5 (Efficient solution) A solution $x \in X$ is said to be efficient(or pareto optimal, nondominated) iff its mapping in the objective space results in a nondominated vector.

The set of all efficient solutions is called efficient(or Pareto optimal) set, and its mapping in the objective space is called Pareto front. A possible approach in multiobjective combinatorial optimization problem solving is to find the minimal set of efficient solutions. But, generating the entire set of pareto optimal solutions is usually, due to the complexity of the underlying problem or to the large number of optima. Therefore, the overall goal is often to identify a good efficient set approximation in stochastic objectives space. These relationships can not compared objec-
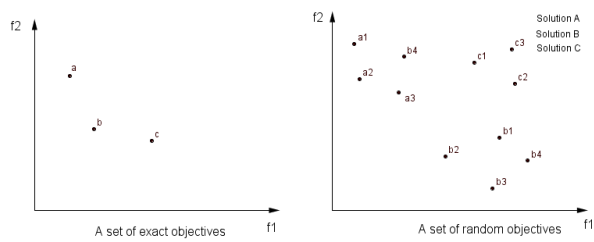

Figure 2. Difference between exact and random solutions

tives value samples $\left\{a_{i}\right\}_{i=1}^{4}$ and $\left\{b_{i}\right\}_{j=1}^{4}$ (Figure2) of given two individuals $x$ and $x^{\prime}$, from this limitation the purpose of this study is to determine stochastic order to describe the relationship among random objectives.

\subsection{Multiobjective Evolutionary Algorithms}

Multiobjective evolutionary algorithms(MOEAs) is a class of stochastic search techniques within metaheuristics that have already been successfully applied to a wide range of optimization problems under uncertainty [21,22]. Research in the design of multi-objective evolutionary algorithms has mainly focused on the fitness assignment, elitism and diversification. We present briefly the notion of these three components:

- Fitness assignment: A notion of Pareto dominance plays an important role to seek Pareto optimality in EMOAs the most example of this component is dominance rank approach.

- Elitism: Is implemented by selecting the best individuals to a mating pool through a such as binary tournament selection of the combined archive and evolving population.

- Diversification: The aim of Diversity maintenance in objective space is to enable a good approximation of the whole Pareto set. An example of diversity strategies are, 
nearest neighbour method and crowding distance.

Pareto multi-objective evolutionary algorithms (PMOEAs) operate on these common principles:

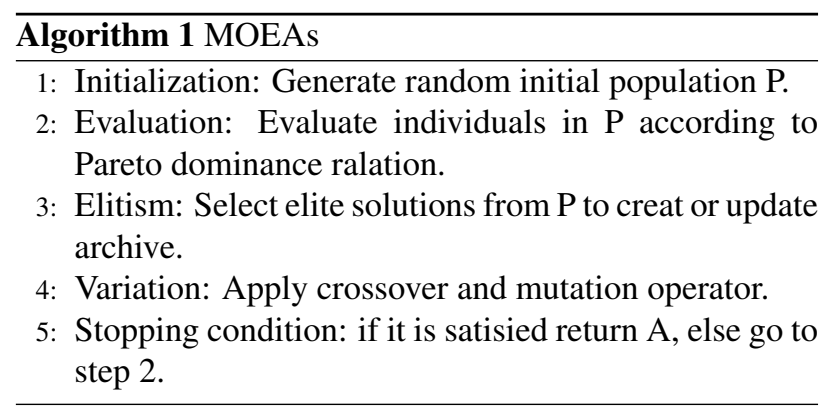

The previous concepts are especially for a deterministic multiobjective case, where the solutions are exact values, we propose in the next section to extended them in stochastic context, by using combinatorial probability.

\section{Probabilistic Approaches in Multi-objective Evolutionary algorithms}

A hybridization of probabilistic programming with metaheuristics have attracted a growing interest during the last few years. This work is part of this research community, the importance of the hybridization proposed in this study is use the probabilistic programming for handling aleatory uncertainty in evolutionary algorithms[24], we choose a conceptually simple approach that is also frequently applied to stochastic programming, namely the optimization based on a fixed sample of random scenarios(i.e, the scenario model is applicable only when the underlying probability distribution is discrete). In this approach, the given distribution of the random vector $\omega$ is approximated by an empirical sample distribution(discrete uniform,binomial ...), obtained by drawing $\mathrm{N}$ scenarios $\left\{\omega_{l}\right\}(l=1, \ldots, N)$, from the original distribution of $\omega$, and replacing the original distribution(continuous or discrete) by a discrete distribution(i.e, A discretization of exponential distribution gives us the binomial distribution) where each of the $\mathrm{N}$ scenarios has the same probability $1 / \mathrm{N}(1 / \mathrm{N}$ in equi-probable sampling). After evaluation technique, the challenging is to estimate the impacts of uncertainty on the samples through a non-parametric statistics: Stochastic order, more specifically probabilistic dominance, then this mathematical instrument is introduced in MOEAs to describe the relationship among random objectives and exploration of the stochastic solutions space. Many stochastic orders [2] exist and can be introduced in MOEAs,the appropriate choice between these relations, is depending on the problem being studied. Briefly, this section is devoted to the application of probabilistic dominance to the with stochastic multi-objective combinatorial programming problems. This way of ranking is based on combinatorial probability. Then the formal definition of elitism and diversification in stochastic context are proposed.

\subsection{The proposed methodology of Probabilistic Optimality}

In the stochastic case, the objective values are different each time a individual is evaluated. So, the vector function $\mathrm{F}$ does not represent a deterministic mapping from the decision space to solution space (Figure.1), because an infinite set of different objective vectors is now assigned to a solution $x \in X$.

In the present work, we choose a conceptually simple approach for probabilistic dominance that compare two stochastic(random) objective vectors.

Definition.6 Given two individual $\mathrm{x}$ and $\mathrm{x}$ ', the probability that solution $F(x, \omega)$ a dominates solution $F\left(x^{\prime}, \omega\right)$ is given by:

$$
\begin{gathered}
x \underset{p r}{ } x^{\prime} \\
\Leftrightarrow \operatorname{Pr}\left(F(x, \omega)<F\left(x^{\prime}, \omega\right)\right)>\operatorname{Pr}\left(F\left(x^{\prime}, \omega\right)<F(x, \omega)\right) \\
\text { where } \operatorname{Pr}\left(F(x, \omega)<F\left(x^{\prime}, \omega\right)\right)= \\
\sum_{i=1}^{l_{1}} \sum_{j=1}^{l_{2}} 1 / l_{1} .1 / l_{2} . I\left(F\left(x, \omega_{i}\right)<F\left(x^{\prime}, \omega_{j}\right)\right)
\end{gathered}
$$

is the non-parametric order relation, and note that (2) is the well known Wilcoxon-Man-Whitney statistic. Which is a non-parametric (i.e., distribution-free) statistical, it takes objective value samples of given two individuals $x$ and $x^{\prime}$, and this probabilistic order which reflects the stochastic nature of the problem in the results.For a perfect ranking function the $\operatorname{Pr}\left(F(x, \omega) \prec F\left(x^{\prime}, \omega\right)\right)$ statistic is 1 and 0 in Incomparable case.

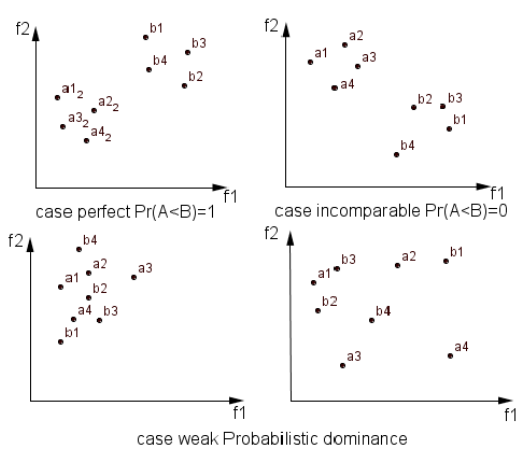

Figure 3. Semantics of Probabilistic Dominance

Within Probabilistic Pareto dominance we can distinguish between Perfect and weak Probabilistic dominance(Figure.3), we propose the properties of this order relation:

- Incompleteness: for two solutions $\mathrm{A}$ and $\mathrm{B}, \operatorname{Pr}(A<$ $B)+\operatorname{Pr}(B<A) \leq 1$

- Weak Probabilistic transitivity: let us consider three solutions $\mathrm{A}, \mathrm{B}$ and $\mathrm{C}$ :

1. if $\operatorname{Pr}(A<B) \leq 0.5$ and $\operatorname{Pr}(B<C) \leq 0.5$ then $\operatorname{Pr}(A<C) \leq 0.5$.

2. if $\operatorname{Pr}(A \prec B)=1$ and $\operatorname{Pr}(B<C)=1$ then $\operatorname{Pr}(A \prec$ $C)=1$. 


\subsection{Fitness assignment scheme}

The main role of this procedure is to guide the search algorithm toward Pareto optimal solutions for a better convergence, but the probabilistic order relation cannot be used to define the layers on solution space, then what is the Rank of each solutions in S?

Definition.7 The rank of each solution $A=$ $\left\{a_{1}, a_{2}, \ldots, a_{l}\right\} \in S$ is determined by the probabilities of other solutions $O=\left\{o_{1}, o_{2}, \ldots, o_{l}\right\} \in S$ dominating it :

$$
\begin{aligned}
& \operatorname{Rank}(A)=\operatorname{Pr}\left(a_{i} \in A\right) \times \prod_{O \neq A}\left(1-\operatorname{Pr}\left(O<a_{i}\right)\right. \\
& \left.\operatorname{Rank}(A)=\frac{1}{l} \sum_{i=1}^{l} \prod_{A \neq O}\left(1-\frac{\left|\left\{o \in O \backslash o<a_{i}\right\}\right|}{|O|}\right)\right)
\end{aligned}
$$

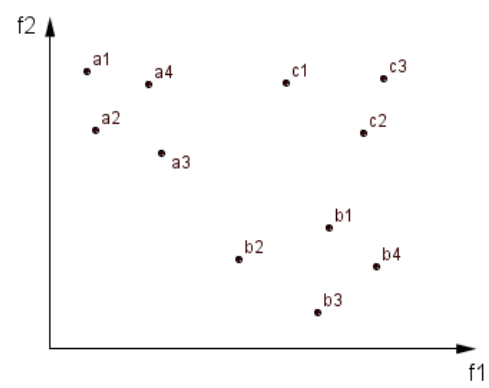

Figure 4. A set of random objectives

$$
\operatorname{Rank}\left(a_{i}\right)=\left(1-\frac{\left|\left\{o \in O \backslash o<a_{i}\right\}\right|}{|Y|}\right)
$$

Example 1: We consider a set of solutions $S=$ $\{A, B, C, D, .$.$\} (Figure.4).$

a- $|A|=4,|C|=3, c_{1}, c_{2}$ and $c_{3}$ are dominated by $a_{3}$, $\operatorname{Pr}(A<C)=0.5 \Rightarrow$ Solution A dominates weakly $\mathrm{C}$.

a- Solution A is non-dominated by any solution $\in S \Rightarrow$ $\operatorname{Rank}(A)=1, \operatorname{similarly} \operatorname{Rank}(B)=1$.

\subsection{Elitism:Probability Memory}

Elitism has always been a major topic of discussion in the evolutionary computation community. In stochastic context, the question of when to accept a newly generated solution despite those previously found, is palliated by the probabilistic memory. The decision to add any solution to the archive is based on a mean probability that the solution is dominated by other solutions in S.

Definition.8 A Probabilistic archiving schema is defined with this relation:

$$
\operatorname{PrArchiv}(p)=\{A \in S \backslash \operatorname{Pr}(A) \geq p\}
$$

where $\operatorname{Pr}(\mathrm{A})=\operatorname{Rank}(\mathrm{A})$ and $p \in[0,1]$ is the fixed probability (chosen by a decision maker) for archiving the best solutions.

\subsection{Diversification}

In stochastic case, diversification is preserved in the resampling procedure. The probability that two solutions are equal is a rare event(for testing $\mathrm{A}=\mathrm{B}$ uses Jaccard similarity coefficient). A few work that is proposed the modification of this technique, for example the crowding distance is a measure of how close an individual is to its neighbors ; large average crowding distance will result in better diversity in the population. We can Define a probabilistic crowding distance [18] as follows:

$$
P C_{i}=\sum_{k=1}^{m} \frac{\mu\left(f_{i+1}^{k}\right)-\mu\left(f_{i-1}^{k}\right)}{\mu\left(f_{\max }^{k}\right)-\mu\left(f_{\min }^{k}\right)}
$$

\subsection{The result of Hybridization:PrMOEA}

The main steps of the Probabilistic Multi-objective Evolutionary Algorithms(PrMOEAs) within an iteration are shown below:

1. Evaluate all solutions in initial population.

2. Fitness assignment: Calculate the probabilistic Pareto ranking of all solutions.

3. Elitism Maintain the best $\mathrm{p} \%$ of the solutions in terms of fitness and discriminate the rest.

4. Perform selection, crossover, and mutation on the population.

We investigate the performance of this algorithm for solving the stochastic version of multi-objective vehicle routing problem.

\section{Case study:Stochastic Multi-objective VRP}

Vehicle routing problem have received increasing attention in recent years. However, in practical situations, the deterministic and single VRP cannot cover real-life contexts, the objectives can be multiple [20] and some components of the VRP are random [25]. In this work, we are interested an important variant of VRP, the so called multiobjective stochastic VRP with two stochastic objectives. A classification of VRP according to the stochastic parameters is proposed in [24,25], and one of the most commonly applied approaches to SVRPs is stochastic programming (SP) where a general introduction is given in [3].

\subsection{Mathematical Formulation}

Let $\mathrm{G}(\mathrm{V}, \mathrm{E})$ be a directed graph, where $\mathrm{E}$ is the set of arcs and $\mathrm{V}$ is the set of nodes: $V=\{0,1, \ldots, n-1, n\}$ represents the $\mathrm{n}$ customers to be visited. A feasible route is represented by the set of served customers, starting and ending at the central depot: $R=\{0,1,2 \ldots n-1, n, 0\}$ for simplicity, the depot is denoted as customer 0 , the depot is treated as the source of service demanded by the customers. The fleet of vehicles, indexed by $k=\{1,2, . ., K\}$ is based at the 
depot, and all the routes must start and end at the depot. The objective of this problems is to find optimal routes with respect to the following two stochastic objectives functions:

$$
\begin{aligned}
& f_{1}(x, \omega)=\sum_{i=0}^{n} \sum_{j=0}^{n} \tilde{T}_{i j} x_{i j}^{k} \\
& f_{2}(x, \omega)=\sum_{i=0}^{n} \sum_{j=0 j \neq i}^{n} \tilde{D}_{i j} x_{i j}^{k}
\end{aligned}
$$

Where $\tilde{T}_{i j}$ is a aleatory duration of travelling from $\mathrm{i}$ to $\mathrm{j}$, the traveling time between two customers $\mathrm{i}$ and $\mathrm{j}$ and $\tilde{D}_{i j}$ is a aleatory travelled distance. These two component are stochastic variables depend on traffic, weather or road conditions or customer requirements such as stochastic demand. The main constraints of MSVRP-SD are the following:

- Vehicle capacity constraint: imposes that the total of stochastic customers' demands for any route must not exceed the limited vehicle capacity.

- Distance constraint: imposes that each vehicle with a limited capacity must deliver goods to the customers with the minimum transportation costs in term of travelled distance. Yet, if the capacity constraint of a vehicle is not satisfied, the delivery fails and causes wasted costs.

In order to resolve and find good solutions for this problem, we propose two probabilistic metaheuristic algorithms: PrMOEAs and PrNSGAII.

\subsection{Solution techniques:PrMOEAs}

To solve MSVRP-SD, we propose a Modified NSGAII. Other contribution in this paper, the NSGAII [9] is adapted to stochastic context by integrating the proposed probabilistic approaches defined in the previous section. PrMOEAs was developed under $\mathrm{C}++$ framework, and PrNSGAII is implemented by modiefied NSGAII under ParadisEO-MOEO [19]. We test a PrNSGAII algorithm on a number of solomon's benchmarks instances: C101, C201, R101, R201 modified. with time windows and stochastic service times and stochastic demands.

Table 1. Parameter Configurations for PrMOEAs

\begin{tabular}{ll}
\hline Parameter & Value \\
\hline Population size & 100 \\
Number of Samples & 25 \\
Max of generation & 500 \\
Crossover rate & 0.9 \\
Mutation rate & 0.2 \\
Probability p & 0.7 \\
\hline
\end{tabular}

Subsequently, we have obtained encouraging results (Figure.6). To evaluate the performance of the algorithm we can compute the means of every stochastic solution and compared it with a solution in deterministic case (Figure.5).

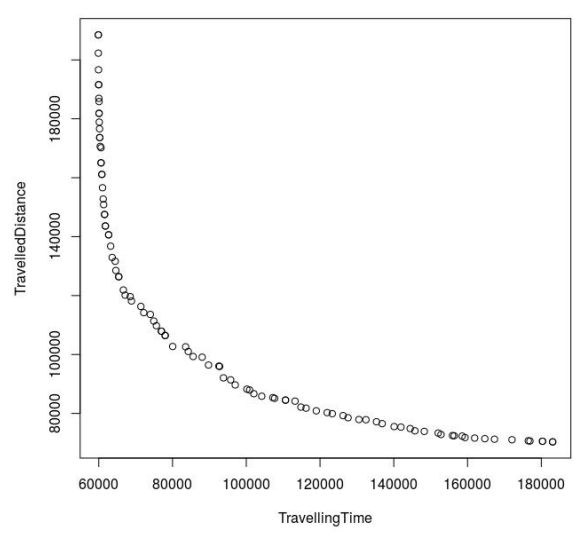

Figure 5. Deterministic Pareto Front(C101-NSGAII)

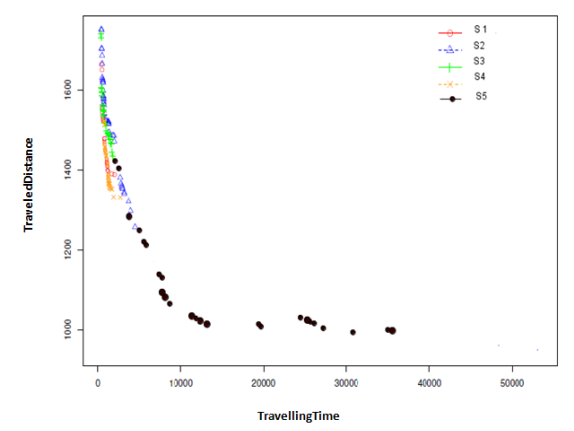

Figure 6. Probabilistic Pareto Front(C101-PrNSGAII)

\section{Conclusion and Discussion}

In this work, an algorithm for stochastic multiobjective combinatorial optimization(SMOCO) problems has been proposed and analyzed. The algorithm is obtained by using a hybridization of probabilistic programming with metaheuristic. Among the main objectives of this hybridization: we extended the dominance criteria, elitism and diversification by using combinatorial probability and non-parametric statistics. The validity of proposed algorithm is empirically investigated and the results demonstrate the capabilitie of this technique to generate a set of well-distributed probabilistic pareto-optimal solutions As future work, it will be also interesting to develop Performance Evaluation, we plan the inclusion of the robustness criteria with Bayesian probability and sensitivity analysis, which aims to judge the quality of stochastic solutions. For testing these novel approaches, the applications can be found in the logistic field, such as Green Transportation problems.

\section{References}

[1] El Ghazali Talbi, Metaheuristics:From Design to Implementation (Wiley, 2009) 624

[2] Moshe Shaked, J. George Shanthikumar, Stochastic Orders (Springer,New York, 2007) 473 
[3] John R. Birge, François Louveaux, Introduction to stochastic programming (Springer, New York, 2011) 500

[4] Walter J. Gutjahr, Two metaheuristics for multiobjective stochastic combinatorial optimization. Stochastic Algorithms:Foundations and Applications. 3777, Lecture Notes in Computer Science, 116-125, (2005)

[5] Norio Baba, Akira Moromito. Three approaches for solving the stochastic multi-objective programming problem. Stochastic Optimization. Lecture Notes in Economics and Mathematical Systems, 379, (Springer, Berlin, Heidelberg),93-109 (1992)

[6] Xinchang Hao, Mitsuo Gen, Lin Lin, Gursel A.Suer. Effective multiobjective EDA for bi-criteria stochastic job-shop scheduling problem. Journal of Intelligent Manufacturing, 1-13, (2015)

[7] Giovanni Petrone, Gianluca Iaccarino, D. Quagliarella. Robustness Criteria in optimisation under uncertainty. Evolutionary and deterministic methods for design, Optimisation and control. CIRA, Capua, Italy 2011

[8] Walter J. Gutjahr, Alois Pichler, Stochastic multiobjective optimization: a survey on non-scalarizing methods, Annals of Operations Research 236, Issue 2, pp 475-499, (January 2016)

[9] Angel A.Juan, Javier Faulin, Scott E.Grasman, Markuts Rabe, Gonçalo Figueira. A review of simheuristics: Extending metaheuristics to deal with stochastic combinatorial optimization problems. Operations Research Perspectives 2, 62-72, (2015)

[10] Kalyanmoy Deb, Samir Agrawal, Amrit Pratap, T Meyarivan, A fast elitist non-dominated sorting genetic algorithm for multi-objective optimization: NSGA-II, Parallel Problem Solving from Nature PPSN VI. Lecture Notes in Computer Science 1917, pp 849-858, (2000)

[11] Josef Schwarz and jiri Ocenasek, Multiobjective bayesian optimization algorithm for combinatorial problems:Theory and practice, Neural Network World 11(5), 423-441, 2001

[12] Yang Liu, Zhi-Ping Fan, Yao Zhang, A method for stochastic multiple criteria decision making based on dominance degrees. Information Sciences 181, 41394153, (2011)

[13] Rajan Filomeno Coelho. Probabilistic Dominance in Multiobjective Reliability-Based Optimization: Theory and Implementation. IEEE Transactions on evolutionary computation 19, No. 2, 214-224, (2015)

[14] Henri Bonnel, Julien Collonge. Optimization over the Pareto outcome set associated with a convex bi- objective optimization problem: theoretical results, deterministic algorithm and application to the stochastic case, Journal of Global Optimization 62, 481-505, (2015)

[15] Henri Bonnel, Julien Collonge.Stochastic Optimization over a Pareto Set Associated with a Stochastic Multi-Objective Optimization Problem. Journal of Global Optimization 162, 405-427, (2014)

[16] Mathieu Basseur, Eckart Zitzler. Handling Uncertainty in Indicator-Based Multiobjective Optimization. International Journal of Computational Intelligence Research 2(3), (2006)

[17] Jörg Fliege, Huifu Xu. Stochastic Multiobjective Optimization: Sample Average Approximation and Applications. Journal of Optimization Theory and Applications 151, Issue 1, pp 135-162, (2011)

[18] Rafael Caballero, Emilio Cerdá, Maria M. Munoz, Lourdes Rey. Stochastic approach versus multiobjective approach for obtaining efficient solutions in stochastic multi-objective programming problems. European Journal of Operational Research 158, 633-648, (2004)

[19] Arnaud Liefooghe, Matthieu Basseur, Laetitia Jourdan, El-Ghazali Talbi. ParadisEO-MOEO: A Framework for Evolutionary Multi-objective Optimization. Evolutionary Multi-Criterion Optimization, Springer, pp 386-400, (2007)

[20] Nicolas Jozefowiez, Frédéric Semet, El-Ghazali Talbi. A Multiobjective vehicle routing problems. European Journal of Operational Research 189,pp 293-309, (2008)

[21] Evan Hughes. Evolutionary multi-objective ranking with uncertainty and noise. In Proceedings of the First Conference on Evolutionary Multi-Criterion Optimization,pp 329-343, (2001)

[22] Kay Chen Tan, Chi Keong Goh. Handling Uncertainties in Evolutionary Multi-Objective Optimization. Computational Intelligence: Research Frontiers. Lecture Notes in Computer Science 5050, pp 262-292, (2008)

[23] Yaochu Jin, J.Branke. Evolutionary Optimization in Uncertain Environments - A Survey. IEEE Transactions on Evolutionary Computation 9(3), 303-317 (2005)

[24] Michel Gendreau, Gilbert Laporte, René Séguin. Stochastic vehicle routing problem, European Journal of Operational Research 88(1), pp 3-12 (1996)

[25] Eshetie Berhan, Birhanu Beshah, Daniel Kitaw. Stochastic Vehicle Routing Problem: A Literature Survey, Journal of Information Knowledge Management 13(3), 12 (2014) 